\title{
PROPAGATION OF A COMBUSTION FRONT IN A STRIATED SOLID MEDIUM: A HOMOGENIZATION ANALYSIS
}

\author{
BY \\ C. M. BRAUNER (Centre de Recherche en Mathématiques de Bordeaux, Talence, France) \\ P. C. FIFE (University of Utah, Salt Lake City, Utah) \\ G. NAMAH (Centre de Recherche en Mathématiques de Bordeaux, Talence, France) \\ AND \\ C. SCHMIDT-LAINÉ (CNRS/Ecole Normale Supérieure de Lyon, Lyon, France)
}

\begin{abstract}
A model problem for the propagation of a combustion front through a periodically inhomogeneous medium is posed. The existence of a steady state solution is proved, in which the front's velocity is periodic in time. Computer simulations are carried out. Finally, through rigorous homogenization techniques, it is shown that when the wavelength of the inhomogeneity is small, the solution may be approximated by a travelling wave solution of the corresponding problem for a medium with certain constant properties.
\end{abstract}

1. Introduction. The propagation of reaction fronts in a striated medium can occur during the combustion of solid material in certain rocket motors. These striations, which consist of thin binder-rich layers, are mainly due to casting processes during fabrication. In this paper we consider the propagation of a flame front in a vertically striated medium, the layers being periodically disposed with a small wavelength $L_{\varepsilon}$ (Fig. 1, p. 468). The horizontal case corresponding to a two-dimensional wave will be treated in a sequel.

Our main purpose is to study the possible qualitative effects that this kind of inhomogeneity might have on the combustion process. The approach followed here is, in addition to computer simulation, that of "mathematical homogenization", in which the front's motion and profile are shown to be approximated by those of a front in a hypothetical homogeneous medium. The properties of this homogeneous approximation are related in an explicit manner to those of the striated one.

A secondary purpose is to set up a homogenization framework in the context of propagative combustion processes. To our knowledge this has not been done

Received August 9, 1991.

1991 Mathematics Subject Classification. Primary 80A25, 35B40.

This work was supported in part through a contract with the Société Nationale des Poudres et Explosifs.

(c) 1993 Brown University 


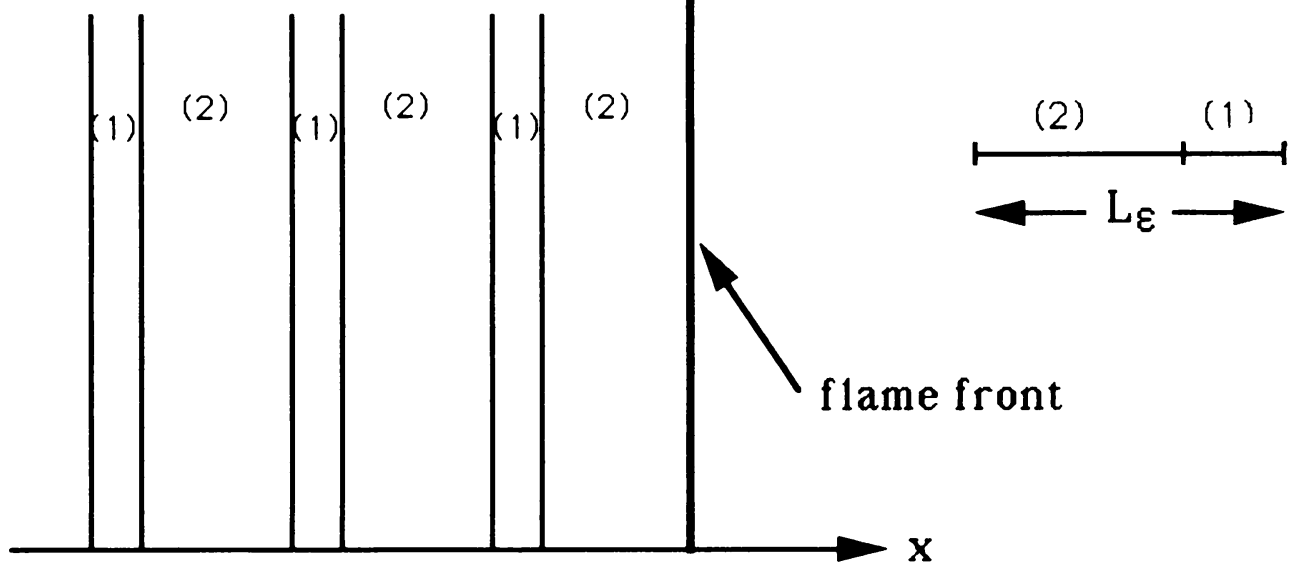

FIG. 1

before, despite the vast number of investigations into principles and techniques of homogenization in other contexts. For this reason especially, we deem it important to present a full rigorous treatment of our problem.

The solid material is characterized by various physical and chemical parameters, which may change according to the type of layer, 1 or 2 (Fig. 1).

First, we set out a one-dimensional Stefan-like model to represent such a propagation. Our model represents the case when the nondimensional activation energy is not too large relative to a nondimensional width of the preheat zone (its ratio to the size of the combusting particles). We then solve the equations numerically for a given wavelength $\varepsilon>0$. The results thus obtained give an indication of the periodicity of the temperature with respect to time. In the fourth section, we show the existence of a solution, which is periodic in time, in a frame fixed to the front. Finally by using homogenization techniques, we show in Sec. 5 that the periodic solution converges to a travelling wave solution with constant velocity when the period tends to zero. An explicit expression is given for the limiting velocity.

2. A 1-D model. For simplicity, we assume the combustion is in the form of a simple exothermic reaction, $A \rightarrow B$, which occurs only on the receding surface of the reactive material.

The material properties of the solid will be characterized by the following five parameters; typical dimensional units are specified.

$d=$ thermal diffusivity in cal/deg-cm-sec

$\rho=$ molar density of the reactant $A$ in moles $/ \mathrm{cm}^{3}$

$\beta=$ heat release in $\mathrm{cal} / \mathrm{mole}$

$c=$ heat capacity in $\mathrm{cal} / \mathrm{deg}-\mathrm{cm}^{3}$

$R(u)=R_{0} K(u)=$ reaction rate function, in moles $/ \mathrm{sec}-\mathrm{cm}^{2}$

Here $u$ is temperature. The area (rather than volume) dependence in the rate function $R$ is due to the fact that we are dealing with a surface reaction. The 
prefactor $R_{0}$ is a fixed number with the given dimensions, and $K(u)$ is a dimensionless Arrhenius factor. The latter is assumed to be a bounded continuous function of temperature, depending on no other small or large parameter; thus the activation energy is not too large.

During steady combustion, the energy that is produced at the receding reactive surface is allocated partly to the gaseous phase behind the combustible material and the remaining part serves to heat the solid. The former portion provides energy needed for thrust, and the latter provides for the chain reaction mechanism by which the reactant ahead of the surface is heated to the temperature needed for its combustion.

We denote by $\theta \quad(0<\theta<1)$ the unknown fraction of energy production that is allocated to the solid phase.

In the striated material considered, the parameters $d, \rho, \beta, c$, and $R$ may depend on the layer type ( 1 or 2$)$. For the purposes of this investigation, however, we examine only the effect of the variation of $d$ (a physical parameter) and $R_{0}$ (a chemical parameter) on the propagation. These parameters will therefore be considered to depend on the spatial coordinate $z$ in a periodic fashion with wavelength $L_{\varepsilon}=\varepsilon Y$. Our basic small parameter is $\varepsilon$. To emphasize their dependence on $\varepsilon$, we write $d=d^{\varepsilon}, R_{0}=R_{0}^{\varepsilon}$.

Let $u^{\varepsilon}=u^{\varepsilon}(x, t)$ denote the temperature, and let the front's position be at $x=$ $\zeta^{\varepsilon}(t)$. The front moves to the left, so that the solid material occupies the halfline $\left\{x<\zeta^{\varepsilon}(t)\right\}$. The equations are as follows:

$$
\begin{gathered}
c \frac{\partial u^{\varepsilon}}{\partial t}-\frac{\partial}{\partial x}\left(d^{\varepsilon} \frac{\partial u^{\varepsilon}}{\partial x}\right)=0, \quad x<\zeta^{\varepsilon}(t) ; \\
\frac{d \zeta^{\varepsilon}}{d t}=-\frac{1}{\rho} R_{0}^{\varepsilon} K\left(u^{\varepsilon}\right), \quad x=\zeta^{\varepsilon}(t) ; \\
d^{\varepsilon} \frac{\partial u^{\varepsilon}}{\partial x}=\theta \beta R_{0}^{\varepsilon} K\left(u^{\varepsilon}\right), \quad x=\zeta^{\varepsilon}(t) ; \\
u^{\varepsilon}(x, t) \rightarrow 0 \text { as } x \rightarrow-\infty .
\end{gathered}
$$

Note that the negative sign in Eq. (2.1b) reflects the fact that the front is moving to the left.

The allocation factor $\theta$ is of course not a given, nor a fixed number, and for transient solutions it will depend on the past history as well. It would be very difficult to calculate from first principles. We consider solutions that deviate by a small amount from being steady travelling waves and so approximate $\theta$ by a constant. The fact that this constant is not known has no bearing on our qualitative results.

For the rest of this paper, it will be convenient to write Eq. (2.1) in a front-fixed frame. This is done by the coordinate transformation: $z=x-\zeta^{\varepsilon}(t)$. We have

$$
\begin{gathered}
c \frac{\partial u^{\varepsilon}}{\partial t}-\frac{\partial}{\partial z}\left(d^{\varepsilon}\left(z+\zeta^{\varepsilon}(t)\right) \frac{\partial u^{\varepsilon}}{\partial z}\right)+c \frac{1}{\rho} R_{0}^{\varepsilon}\left(\zeta^{\varepsilon}(t)\right) K\left(u^{\varepsilon}(0, t)\right) \frac{\partial u^{\varepsilon}}{\partial z}=0, \quad z<0 \\
\frac{d \zeta^{\varepsilon}}{d t}=-\frac{1}{\rho} R_{0}^{\varepsilon}\left(\zeta^{\varepsilon}(t)\right) K\left(u^{\varepsilon}(0, t)\right)
\end{gathered}
$$




$$
\begin{gathered}
d^{\varepsilon}\left(\zeta^{\varepsilon}(t)\right) \frac{\partial u^{\varepsilon}}{\partial z}=\theta \beta R_{0}^{\varepsilon}\left(\zeta^{\varepsilon}(t)\right) K\left(u^{\varepsilon}\right), \quad z=0 ; \\
u^{\varepsilon}(z, t) \rightarrow 0 \quad \text { as } z \rightarrow-\infty
\end{gathered}
$$

Hence forward, we consider this system of equations.

3. Numerical resolution. We must first specify initial conditions for the two unknowns $\left(u^{\varepsilon}, \zeta^{\varepsilon}\right)$. We take

$$
\begin{gathered}
\zeta^{\varepsilon}(0)=0, \\
u^{\varepsilon}(z, 0)=u_{0}(z),
\end{gathered}
$$

where $u_{0}$ is of exponential type. In fact, we take $u_{0}$ to be the steady state solution of the homogeneous problem (where $d^{\varepsilon}$ and $R_{0}^{\varepsilon}$ are constants). It is given by

$$
u_{0}(z)=\frac{\theta \beta \rho}{c} \exp \left(-\frac{c \zeta_{t}}{d} z\right), \quad z<0,
$$

where

$$
\zeta_{t}=-\rho^{-1} R_{0} K(\theta \beta \rho / c)
$$

Figures $2 \mathrm{a}-\mathrm{c}$ show the results obtained by the use of a finite difference scheme. The right-hand side curves show temperature against time plots at the front (the uppermost curve) and at two other values of $z(z=-0.5$ and -1.). The curves on the left represent temperature against distance plots for different values of time, including the initial condition.

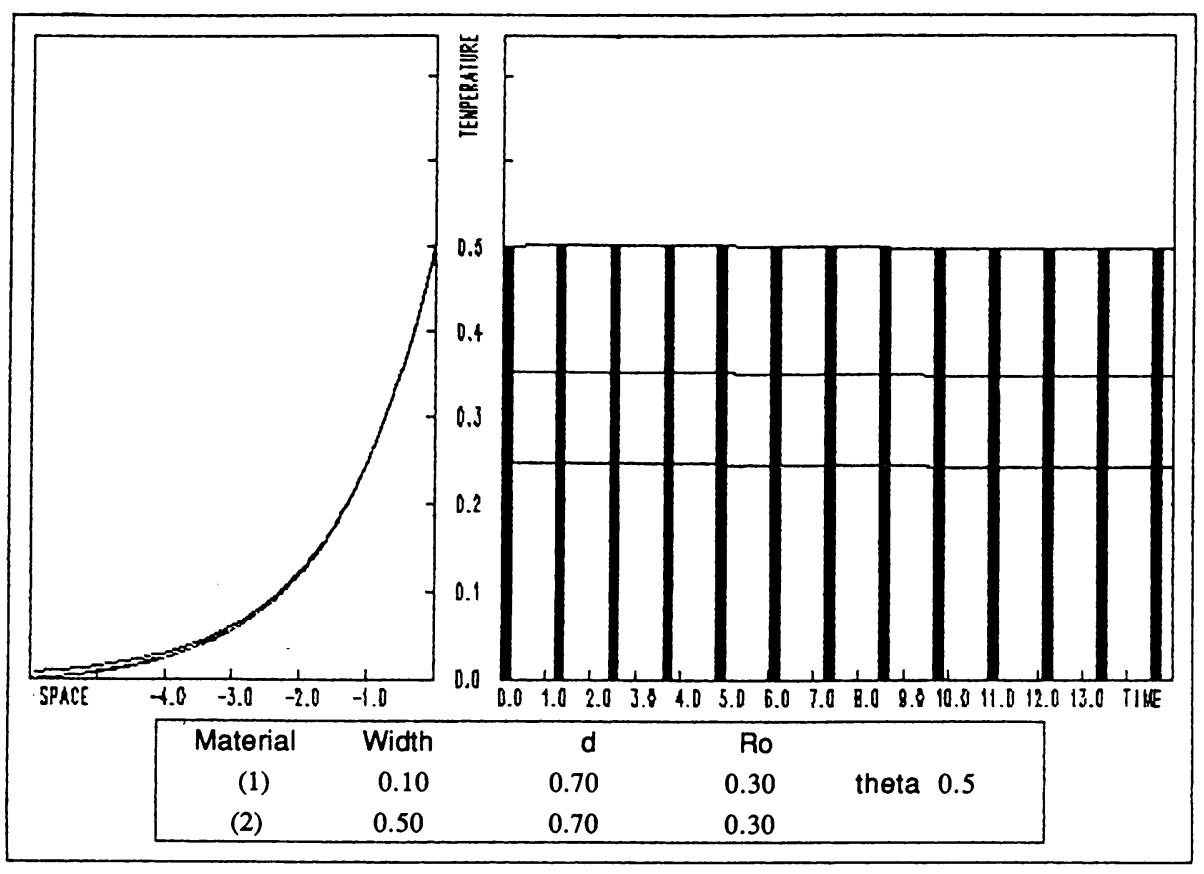

FIG. 2a. Homogeneous medium 


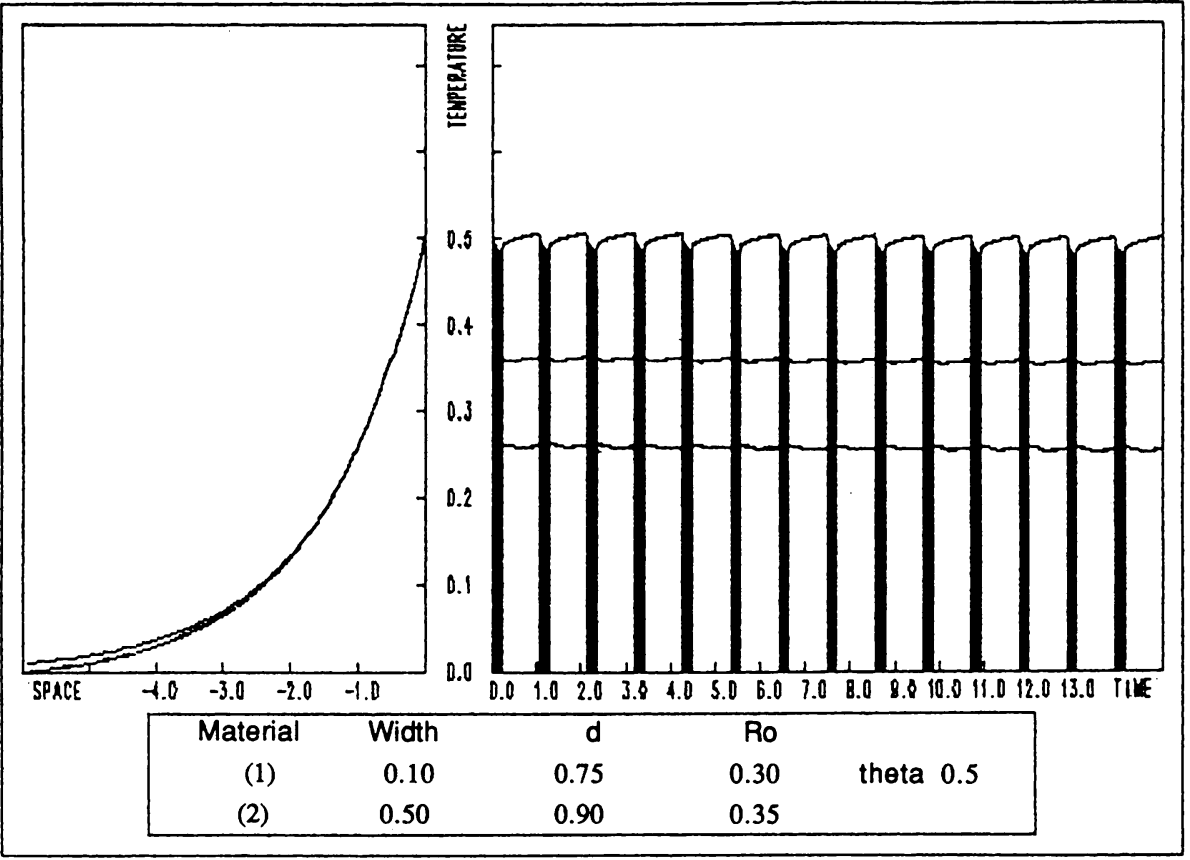

FIG. 2b. Slightly heterogeneous medium

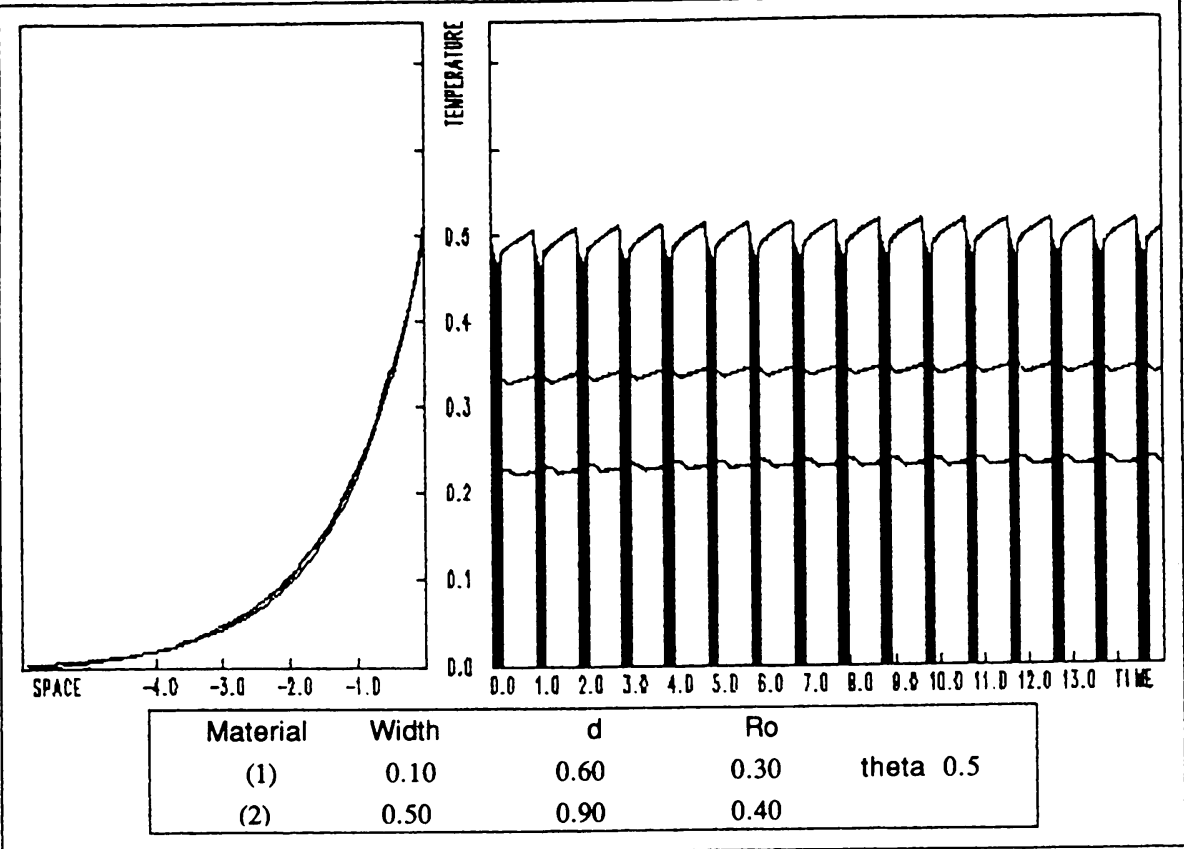

FIG. 2c. Heterogeneous medium 
Figure $2 \mathrm{a}$ corresponds to a homogeneous medium $\left(d_{1}=d_{2}, R_{01}=R_{02}\right)$. As expected, the temperature curves are independent of time.

Figure $2 \mathrm{~b}$ corresponds to a heterogeneous medium. The established temperature profiles are periodic in time. The curve at $z=-1.0$ seems almost to be a constant. This is due to the fact that the heterogeneous character of the medium is not so important. For a more pronounced heterogeneity, we get profiles as shown in Fig. 2c.

The temperature profiles thus obtained bring us to look for the existence of a periodic solution for the system (2.2).

\section{Existence of a periodic solution.}

4.1. Existence theorem. Consider the following problem for a fixed positive $\varepsilon$ :

$$
\begin{gathered}
c \frac{\partial u^{\varepsilon}}{\partial t}-\frac{\partial}{\partial z}\left(d^{\varepsilon}\left(z+\zeta^{\varepsilon}(t)\right) \frac{\partial u^{\varepsilon}}{\partial z}\right)+c \frac{1}{\rho} R_{0}^{\varepsilon}\left(\zeta^{\varepsilon}(t)\right) K\left(u^{\varepsilon}(0, t)\right) \frac{\partial u^{\varepsilon}}{\partial z}=0, \\
\frac{d \zeta^{\varepsilon}}{d t}=-\frac{1}{\rho} R_{0}^{\varepsilon}\left(\zeta^{\varepsilon}(t)\right) K\left(u^{\varepsilon}(0, t)\right),
\end{gathered}
$$

subject to the boundary conditions

$$
\begin{gathered}
d^{\varepsilon}\left(\zeta^{\varepsilon}(t)\right) \frac{\partial u^{\varepsilon}}{\partial z}=\theta \beta R_{0}^{\varepsilon}\left(\zeta^{\varepsilon}(t)\right) K\left(u^{\varepsilon}\right), \quad z=0, \\
u^{\varepsilon}(z, t) \rightarrow 0 \quad \text { as } z \rightarrow-\infty,
\end{gathered}
$$

and the initial and periodicity conditions

$$
\begin{gathered}
\zeta^{\varepsilon}(0)=0, \\
u^{\varepsilon}(z, 0)=u^{\varepsilon}\left(z, T^{\varepsilon}\right),
\end{gathered}
$$

with $T^{\varepsilon}$ defined as the time taken by the front to travel a distance of one period in space, i.e.,

$$
L_{\varepsilon}=\int_{0}^{T^{\varepsilon}} \rho^{-1} R_{0}^{\varepsilon}\left(\zeta^{\varepsilon}(t)\right) K\left(u^{\varepsilon}(0, t)\right) d t .
$$

The unknowns of this problem are the functions $u^{\varepsilon}=u^{\varepsilon}(z, t), \zeta^{\varepsilon}=\zeta^{\varepsilon}(t)$, and the period $T^{\varepsilon}$.

Let us now assume the following:

$d^{\varepsilon}$ and $R_{0}^{\varepsilon}$ are positive, continuous, bounded, and periodic of period $L_{\varepsilon}$ in the space variable;

$K(u)$ is a positive, continuous, and bounded real function.

By hypothesis, we have estimates of the form

$$
\begin{aligned}
& 0<\underline{R} \leq R_{0}^{\varepsilon} \leq \bar{R}, \\
& 0<\underline{d} \leq d^{\varepsilon} \leq \bar{d}, \\
& 0<\underline{K} \leq K \leq \bar{K} .
\end{aligned}
$$


Then by the definition of $T^{\varepsilon}$, we deduce

$$
T_{\min } \leq T^{\varepsilon} \leq T_{\max }
$$

with

$$
T_{\min }=\frac{\rho L_{\varepsilon}}{\bar{R} \bar{K}}, \quad T_{\max }=\frac{\rho L_{\varepsilon}}{\underline{R} \underline{K}} .
$$

In what follows, we are going to use the following notation:

$$
\Omega=(-\infty, 0), \quad H=L^{2}(\Omega), \quad V=H^{1}(\Omega) ;
$$

$|\cdot|$ and $\|\cdot\|$ represent the norms in $H$ and $V$ respectively, and $(\cdot)$ denotes the scalar product in $H$ and the duality product in $V, V^{\prime}$.

THeOREM 4.1. Under the assumptions (4.5), (4.6), there exists a solution $\left(u^{\varepsilon}, \zeta^{\varepsilon}, T^{\varepsilon}\right)$ to the problem (4.1)-(4.4) with

$$
\begin{gathered}
u^{\varepsilon} \in L^{2}\left(0, T^{\varepsilon} ; V\right) \cap L^{\infty}\left(0, T^{\varepsilon} ; H\right), \\
\zeta^{\varepsilon} \in W^{1, \infty}\left(0, T^{\varepsilon}\right), \quad T^{\varepsilon}>0,
\end{gathered}
$$

such that $u^{\varepsilon}$ is $T^{\varepsilon}$-periodic in time.

4.2. A positivity lemma. Suppose that Theorem 4.1 holds. The following lemma then follows.

LEMMA 4.1. The solution $u^{\varepsilon}$ is positive.

Proof. We use the weak maximum principle. We multiply the equation in $u^{\varepsilon}$ by $\left(u^{\varepsilon}\right)^{-}$and integrate over $\Omega$. This can be justified as in Lions [5, p. 290]. Letting $K$ stand for $K\left(u^{\varepsilon}(0, t)\right)$, we have

$$
0=-\frac{1}{2} c \frac{d}{d t}\left|u^{\varepsilon-}\right|^{2}+c \rho^{-1} R_{0}^{\varepsilon} K \int_{-\infty}^{0} \frac{\partial u^{\varepsilon}}{\partial z} u^{\varepsilon-} d z-\int_{-\infty}^{0} \frac{\partial}{\partial z}\left(d^{\varepsilon} \frac{\partial u^{\varepsilon}}{\partial z}\right) u^{\varepsilon-} d z,
$$

i.e.,

$$
-\frac{1}{2} c \frac{d}{d t}\left|u^{\varepsilon-}\right|^{2}-\frac{1}{2} c \rho^{-1} R_{0}^{\varepsilon} K u^{\varepsilon-}(0, t)-\int_{-\infty}^{0} d^{\varepsilon}\left(\frac{\partial u^{\varepsilon-}}{\partial z}\right)^{2} d z-\left[d^{\varepsilon} \frac{\partial u^{\varepsilon}}{\partial z} u^{\varepsilon-}\right]_{-\infty}^{0}=0 .
$$

Since $\left.d^{\varepsilon} \frac{\partial u^{\varepsilon}}{\partial z}\right|_{x=0}=\theta \beta R_{0}^{\varepsilon} K$, we finally have

$$
-\frac{1}{2} c \frac{d}{d t}\left|u^{\varepsilon-}\right|^{2}-\frac{1}{2} c \rho^{-1} R_{0}^{\varepsilon} K u^{\varepsilon-}(0, t)-\int_{-\infty}^{0} d^{\varepsilon}\left(\frac{\partial u^{\varepsilon-}}{\partial z}\right)^{2} d z-\theta \beta R_{0}^{\varepsilon} K u^{\varepsilon-}(0, t)=0 .
$$

We now integrate in time over one period to obtain

$$
-\frac{1}{2} \int_{0}^{T^{\varepsilon}} c \frac{d}{d t}\left|u^{\varepsilon-}\right|^{2} d t-\int_{0}^{T^{\varepsilon}} \Lambda(t) d t=0
$$

where

$$
\Lambda(t)=\frac{1}{2} c \rho^{-1} R_{0}^{\varepsilon} K u^{\varepsilon-}(0, t)+\int_{-\infty}^{0} d^{\varepsilon}\left(\frac{\partial u^{\varepsilon-}}{\partial z}\right)^{2} d z+\theta \beta R_{0}^{\varepsilon} K u^{\varepsilon-}(0, t) \geq 0 .
$$


Since $u^{\varepsilon}$ is periodic, the first term vanishes, hence $\Lambda \equiv 0$, which leads to $u^{\varepsilon-} \equiv 0$. This completes the proof of Lemma 4.1.

4.3. A normalized problem. Since $\varepsilon$ is fixed for the rest of this section, its symbol will be dropped. Let us then normalize in time by setting

$$
t=s T
$$

in the problem (4.1)-(4.4). The latter becomes

$$
\begin{gathered}
c \frac{\partial u}{\partial s}-T \frac{\partial}{\partial z}\left(d \frac{\partial u}{\partial z}\right)+c T \rho^{-1} R_{0} K(u(0, s)) \frac{\partial u}{\partial z}=0, \quad z<0, \\
\zeta(s)=-T \int_{0}^{s} \rho^{-1} R_{0}(\zeta(\sigma)) K(u(0, \sigma)) d \sigma
\end{gathered}
$$

with the periodicity condition

$$
u(z, 0)=u(z, 1)
$$

and the boundary conditions

$$
\begin{gathered}
d(\zeta(s)) \frac{\partial u}{\partial z}=\theta \beta R_{0}(\zeta(s)) K(u), \quad z=0, \\
u(z, s) \rightarrow 0 \quad \text { as } z \rightarrow-\infty .
\end{gathered}
$$

Remark 4.1. The problems given by Eqs. (4.1)-(4.4) and (4.10)-(4.13) are equivalent. Therefore proving Theorem 4.1 is reduced to proving the following theorem.

THEOREM 4.2. Under the assumptions of Theorem 4.1, there exists a solution $(u, \zeta, T)$ to the problem (4.10)-(4.13) with

$$
\begin{gathered}
u \in L^{2}(0,1 ; V) \cap L^{\infty}(0,1 ; H), \\
\zeta \in W^{1, \infty}(0,1) \text { and } T>0 .
\end{gathered}
$$

The rest of this section will be devoted to proving Theorem 4.2.

4.4. Proof of Theorem 4.2. We are going to proceed according to the following steps. First, the problem is posed on a bounded interval $\Omega_{A}=(-A, 0), A>0$, on which we prove the existence of an approximate solution $\left(u_{m}(t), \zeta_{m}(t)\right)$ for $t \in[0,1]$ (Faedo-Galerkin method). The existence of the period is also proved by the use of a fixed point result in finite dimensions (Brouwer's theorem). Then by writing down the necessary estimates, we pass to the limit in the dimension $(m \rightarrow \infty)$. The proof is completed by a passage to the limit as the interval length $A \rightarrow \infty$.

4.4.1. An approximate Cauchy problem. Let

$$
\begin{gathered}
V_{A}=\left\{v \in H^{1}\left(\Omega_{A}\right), v(-A)=0\right\}, \quad H_{A}=L^{2}\left(\Omega_{A}\right), \\
|\cdot|_{A}=\text { norm in } H_{A}, \quad\|\cdot\|_{A}=\text { norm in } V_{A}, \\
(u, v)_{A}=\text { the scalar product in } H_{A} \text { and the duality product in }\left(V_{A}, V_{A}^{\prime}\right) .
\end{gathered}
$$

By considering a basis of $V, w_{1}, \ldots, w_{m}, \ldots$, we look for an approximate solution $u_{m}$ of the form

$$
u_{m}(s)=\sum_{i=1}^{m} g_{i}^{m}(s) w_{i}
$$


satisfying

$$
\begin{gathered}
\left(c \frac{\partial u_{m}}{\partial s}, w_{j}\right)_{A}+a_{m}\left(u_{m}(s), w_{j}\right)=\left(L(s), w_{j}\right)_{A}, \quad 1 \leq j \leq m \\
\frac{d \zeta_{m}}{d s}=-T \rho^{-1} R_{0}\left(\zeta_{m}(s)\right) K\left(u_{m}(0, s)\right) \\
u_{m}(0)=u_{0 m} \in W_{m}=\left[w_{1}, \ldots, w_{m}\right] \\
\zeta_{m}(0)=0
\end{gathered}
$$

with

$$
\begin{gathered}
a_{m}(u, v)=T \int_{\Omega} d_{m} \frac{\partial u}{\partial z} \frac{\partial v}{\partial z} d z+c T \int_{\Omega} \rho^{-1} R_{0 m} K(u(0, s)) \frac{\partial u}{\partial z} v d z \\
(L(s), v)_{A}=\theta \beta R_{0 m} K(u(0, s)) v(0) \quad \forall v \in V_{A}, \\
d_{m}=d\left(z+\zeta_{m}(s)\right), \quad R_{0 m}=R_{0}\left(\zeta_{m}(s)\right) .
\end{gathered}
$$

The existence of $\left(u_{m}, \zeta_{m}\right)$ on some interval $\left[0, t_{m}\right]$ follows from standard results on the existence of solutions of systems of nonlinear ordinary differential equations. That $t_{m}=1$ will be deduced from a priori estimates on $u_{m}$.

4.4.2. Estimates on $u_{m}$. The first energy type equality is obtained by multiplying Eq. (4.14) by $g_{j}^{m}$ and summing those relations for $j=1, \ldots, m$. We obtain

$$
\left(c \frac{\partial u_{m}}{\partial s}, u_{m}\right)_{A}+a_{m}\left(u_{m}(s), u_{m}(s)\right)=\left(L(s), u_{m}(s)\right)_{A},
$$

i.e.,

$$
\begin{aligned}
& \frac{1}{2} c \frac{d}{d s}\left|u_{m}(s)\right|^{2}+T \int_{\Omega} d_{m}\left(\frac{\partial u_{m}}{\partial z}(s)\right)^{2}+\frac{1}{2} c T \rho^{-1} R_{0} K\left(u_{m}(0, s)\right) u_{m}^{2}(0, s) \\
& \quad=\theta \beta R_{0} K\left(u_{m}(0, s)\right) u_{m}(0, s)
\end{aligned}
$$

By choosing an $\alpha>0$ such that $T \rho^{-1} R_{0} \geq \theta \alpha$ and writing

$$
u_{m}(0, s) \leq \frac{\alpha}{2} u_{m}^{2}(0, s)+\frac{1}{2 \alpha}
$$

we get by using Eq. (4.7)

$$
\frac{1}{2} \frac{d}{d s}\left|u_{m}(s)\right|_{A}^{2}+C_{1}\left|\frac{\partial u_{m}}{\partial z}\right|_{A}^{2} \leq C_{2}
$$

where here and in what follows the $C$ 's are positive constants independent of $m$.

Thus for all $s \in\left[0, t_{m}\right]$, we have

$$
\left|u_{m}(s)\right|_{A}^{2}+2 C_{1} \int_{0}^{s}\left|\frac{\partial u_{m}}{\partial z}(\sigma)\right|_{A}^{2} d \sigma \leq\left|u_{0 m}\right|_{A}^{2}+2 C_{2} t_{m} \leq C_{3} .
$$

Inequality (4.21) then gives

$$
\left|u_{m}(s)\right|_{A}^{2} \leq C_{3}
$$

Note that Eq. (4.22) holds for all $s \in\left[0, t_{m}\right]$ with a bound independent of $m$; therefore, we can take $t_{m}=1$. 
From Eqs. (4.21) and (4.22) we have

$$
u_{m} \text { is bounded in } L^{\infty}\left(0,1 ; H_{A}\right) \cap L^{2}\left(0,1 ; V_{A}\right) .
$$

Let $\mathscr{A}_{m} \in \mathscr{L}\left(V, V^{\prime}\right)$, the operator associated with the bilinear form $a_{m}$. It is easily shown that the following inequality holds:

$$
\left\|\mathscr{A}_{m} u_{m}(s)\right\|_{V_{A}^{\prime}} \leq C_{4}\left(\left\|u_{m}(s)\right\|_{A}+1\right) \text {. }
$$

From Eqs. (4.23) and (4.24), we then have

$$
\mathscr{A}_{m} u_{m} \text { bounded in } L^{2}\left(0,1 ; V_{A}^{\prime}\right)
$$

which finally leads to

$$
\frac{\partial u_{m}}{\partial s} \text { bounded in } L^{2}\left(0,1 ; V_{A}^{\prime}\right) .
$$

4.4.3. Estimates on $\zeta_{m}$. We have $d \zeta_{m} / d s=-T \rho^{-1} R_{0} K\left(u_{m}(0, s)\right)$.

Since $R_{0}$ and $K$ are bounded [see Eq. (4.7)], we have

$$
\frac{d \zeta_{m}}{d s} \text { bounded in } L^{\infty}(0,1)
$$

Now $\zeta_{m}$ is given by

$$
\zeta_{m}(s)=-T \rho^{-1} \int_{0}^{s} R_{0} K\left(u_{m}(0, \sigma)\right) d \sigma
$$

and therefore

$$
\zeta_{m} \text { bounded in } W^{1, \infty}(0,1)
$$

4.4.4. Existence of the period (fixed point). Let us consider the following mapping for $m$ fixed:

with

$$
\begin{gathered}
\Phi: W_{m} \times(0,+\infty) \rightarrow W_{m} \times(0,+\infty), \\
\left(u_{0 m}, T\right) \rightarrow\left(\hat{u}_{0 m}, \widehat{T}_{m}\right),
\end{gathered}
$$

$$
\begin{gathered}
u_{0 m}=u_{m}(0), \quad \hat{u}_{0 m}=u_{m}(1), \\
\widehat{T}_{m}=L / \int_{0}^{1} \rho^{-1} R_{0} K\left(u_{m}(0, s)\right) d s .
\end{gathered}
$$

Let us suppose that the following lemma holds.

LEMMA 4.1. (i) $\Phi$ is a continuous mapping from $W_{m} \times(0,+\infty)$ onto itself, and

(ii) $\exists C>0$ independent of $m$ such that

$$
\left|u_{0 m}\right| \leq C, T_{\min } \leq T \leq T_{\max } \text { implies }\left|\hat{u}_{0 m}\right| \leq C, T_{\min } \leq \widehat{T}_{m} \leq T_{\max } .
$$

Then by applying Brouwer's fixed point theorem we obtain the existence of $\left(u_{0 m}, T_{m}\right)$ such that

$$
\Phi\left(u_{0 m}, T_{m}\right)=\left(u_{0 m}, T_{m}\right) .
$$

Now we prove Lemma 4.1.

Proof of Lemma 4.1. (i) To show that $\Phi$ is continuous, take a sequence $\left(u^{n}, T^{n}\right) \in$ $\left(W_{m} \times(0, \infty)\right)$ that converges to $\left(u_{0 m}, T\right)$. It suffices then to show that $\phi\left(u^{n}, T^{n}\right)$ 
tends to $\left(\hat{u}_{0 m}, \widehat{T}_{m}\right)$ when $n$ goes to infinity. The estimates $(4.23)-(4.27)$ hold for $\left(u^{n}, \zeta^{n}\right)$, which is the solution corresponding to the given data $\left(u^{n}, T^{n}\right) . \widehat{T}_{n}$ is then given by

$$
\widehat{T}^{n}=L / \int_{0}^{1} \rho^{-1} R_{0}^{n} K\left(u^{n}(0, s)\right) d s, \quad R_{0}^{n}=R_{0}\left(\zeta^{n}(s)\right) .
$$

Finally by extracting a subsequence, still denoted by $\left(u^{n}, \widehat{T}^{n}\right)$, it is straightforward to check that

$$
\left(u^{n}(1), \widehat{T}^{n}\right) \rightarrow\left(\hat{u}_{0 m}, \widehat{T}_{m}\right) \text { as } n \rightarrow+\infty .
$$

(ii) For the second part of the lemma, we only have to show the existence of $C$. Suppose $\left|u_{0 m}\right|_{A} \leq C$. From Eq. (4.20), we have

$$
\frac{d}{d s}\left|u_{m}(s)\right|_{A}^{2}+C_{1}\left|\frac{\partial u_{m}}{\partial z}\right|_{A}^{2} \leq C_{2}
$$

Poincaré's inequality now gives

$$
\left|\frac{\partial u_{m}}{\partial z}(s)\right|_{A}^{2} \geq C_{5}\left|u_{m}(s)\right|_{A}^{2}
$$

Eqs. (4.35) and (4.36) then yield

$$
\frac{d}{d s}\left|u_{m}(s)\right|_{A}^{2}+C_{6}\left|u_{m}(s)\right|_{A}^{2} \leq C_{2} .
$$

We then multiply Eq. (4.37) by $\exp \left(C_{6} s\right)$ and integrate in time between 0 and 1 . We have

$$
\left|u_{m}(1)\right|_{A}^{2} \leq e^{-C_{6}}\left|u_{0 m}\right|_{A}^{2}+C_{7} .
$$

Then by taking $C^{2} \geq C_{7} /\left(1-e^{-C_{6}}\right)$, we have

$$
\left|\hat{u}_{0 m}\right|_{A} \leq C \text {. }
$$

This completes the proof of Lemma 4.1.

4.4.5. Passage to the limit $(m \rightarrow+\infty)$. Let us recall that so far we have the existence of $\left(u_{m}, \zeta_{m}, T_{m}\right)$, a solution of

$$
c \frac{\partial u_{m}}{\partial s}-T_{m} \frac{\partial}{\partial z}\left(d_{m} \frac{\partial u_{m}}{\partial z}\right)+c T_{m} \rho^{-1} R_{0 m} K\left(u_{m}(0, s)\right) \frac{\partial u_{m}}{\partial z}=0, \quad z \in \Omega_{A}
$$

(in the weak sense: the left side is orthogonal to each $w_{j}$ in $W_{m}$ ),

$$
\frac{d \zeta_{m}}{d s}=-T_{m} \rho^{-1} R_{0}\left(\zeta_{m}\right) K\left(u_{m}(0, s)\right)
$$

subject to the periodic and initial conditions

$$
u_{m}(0)=u_{m}(1), \quad \zeta_{m}(0)=0,
$$

with $T_{m}$ given by

$$
T_{m}=L / \int_{0}^{1} \rho^{-1} R_{0 m} K\left(u_{m}(0, s)\right) d s
$$


We now use the estimates (4.24)-(4.29). We extract subsequences from $\left(\zeta_{m}\right)$ and $\left(u_{m}\right)$ and denote their limits by $\zeta_{A}$ and $u_{A}$. We then have

$$
\begin{aligned}
\zeta_{m} & \rightarrow \zeta_{A} \text { in } L^{\infty}(0,1) \text { weak star }, \\
\frac{d \zeta_{m}}{d s} & \rightarrow \frac{d \zeta_{A}}{d s} \text { in } L^{\infty}(0,1) \text { weak star. }
\end{aligned}
$$

By compactness, we have then

$$
\zeta_{m} \rightarrow \zeta_{A} \text { uniformly on }[0,1] \text {. }
$$

Since $R_{0}$ and $d$ are continuous, we also have by Lebesgue's theorem

$$
\begin{array}{r}
R_{0 m}=R_{0 m}\left(\zeta_{m}\right) \rightarrow R_{0}\left(\zeta_{A}\right) \quad \text { in } L^{p}(0,1), \quad 1 \leq p<\infty, \\
d_{m}=d\left(z+\zeta_{m}\right) \rightarrow d\left(z+\zeta_{A}\right) \quad \text { in } L^{p}\left(\Omega_{A} \times(0,1)\right), \quad 1 \leq p<\infty .
\end{array}
$$

From Eq. (4.45), we have

$$
\zeta_{m}(0) \rightarrow \zeta_{A}(0)
$$

Concerning $u_{m}$, we have

$$
\begin{aligned}
u_{m} & \rightarrow u_{A} \quad \text { weakly in } L^{2}\left(0,1 ; V_{A}\right), \\
u_{m} & \rightarrow u_{A} \quad \text { strongly in } L^{2}\left(0,1 ; H_{A}\right), \\
\frac{\partial}{\partial z}\left(d_{m} \frac{\partial u_{m}}{\partial z}\right) & \rightarrow \frac{\partial}{\partial z}\left(d_{A} \frac{\partial u_{A}}{\partial z}\right) \quad \text { weakly in } L^{2}\left(0,1 ; V_{A}^{\prime}\right), \\
\frac{\partial u_{m}}{\partial s} & \rightarrow \frac{\partial u_{A}}{\partial s} \quad \text { weakly in } L^{2}\left(0,1 ; V_{A}^{\prime}\right) .
\end{aligned}
$$

From Eqs. (4.48) and (4.51), we have

$$
\begin{aligned}
& u_{m}(0) \rightarrow u_{A}(0) \quad \text { weakly in } H_{A}, \\
& u_{m}(1) \rightarrow u_{A}(1) \quad \text { weakly in } H_{A} .
\end{aligned}
$$

Since $u_{m}(0)=u_{m}(1)$, we have

$$
u_{A}(0)=u_{A}(1)
$$

By denoting the limit of $T_{m}$ by $T_{A}$, we have

$$
T_{m} \rightarrow T_{A} \text { as } m \rightarrow+\infty \text {. }
$$

To be able to pass to the limit in Eqs. (4.40)-(4.43), we still have to write down the limit of $K\left(u_{m}(0, s)\right)$.

\section{LEMMA 4.2.}

$$
K\left(u_{m}(0, \cdot)\right) \rightarrow K\left(u_{A}(0, \cdot)\right) \quad \text { as } m \rightarrow \infty \text {, a.e. and in } L^{p}(0,1), 1 \leq p<\infty .
$$

Proof. First, we show that

$$
\left.u_{m} \rightarrow u_{A} \quad \text { strongly in } L^{2}\left(0,1 ; H^{s}\left(\Omega_{A}\right)\right) \text { for } s \in\right] \frac{1}{2}, 1[\text {. }
$$

In fact, let $W_{A}=\left\{v: v \in L^{2}\left(0,1 ; V_{A}\right), \partial v / \partial s \in L^{2}\left(0,1 ; V_{A}^{\prime}\right\}\right.$. Then by a compactness lemma (see, e.g., Lions [5]), we have

$$
W_{A} \in L^{2}\left(0,1 ; H^{s}\left(\Omega_{A}\right)\right) \text { with a compact embedding, } \frac{1}{2}<s<1 \text {. }
$$


Then Eq. (4.56) is derived from Eqs. (4.48) and (4.51). Next, by using a trace theorem, we deduce that

$$
u_{m}(0, \cdot) \rightarrow u_{A}(0, \cdot) \text { in } L^{2}(0,1) \text { strongly. }
$$

Finally, Lemma 4.2 is obtained by the continuity of the function $K$ and Lebesgue's theorem.

We can thus pass to the limit in the approximate problem.

4.4.6. Passage to the limit $A \rightarrow \infty$. The limit $\left(u_{A}, \zeta_{A}, T_{A}\right)$ obtained in the previous section depends on the interval $\Omega_{A}=(-A, 0)$. We will now let $A$ approach infinity. We consider a sequence $A_{n}$ tending to infinity as $n \rightarrow \infty$ and then a corresponding sequence of solutions on the interval $\left(-A_{n}, 0\right)$, denoted by $\left(u_{n}, \zeta_{n}, T_{n}\right)$, which satisfy

$$
\begin{gathered}
c \frac{\partial u_{n}}{\partial s}-T_{n} \frac{\partial}{\partial z}\left(d_{n} \frac{\partial u_{n}}{\partial z}\right)+c \rho^{-1} T_{n} R_{0}\left(\zeta_{n}\right) K\left(u_{n}(0, s)\right) \frac{\partial u_{n}}{\partial z}=0, \\
\frac{d \zeta_{n}}{d s}=-T_{n} \rho^{-1} R_{0}\left(\zeta_{n}\right) K\left(u_{n}(0, s)\right),\left(-A_{n}, 0\right) \\
u_{n}(z, 0)=u_{n}(z, 1) \\
\zeta_{n}(0)=0
\end{gathered}
$$

subject to the boundary conditions

$$
\begin{gathered}
d_{n} \frac{\partial u_{n}}{\partial z}=\theta \beta R_{0}\left(\zeta_{n}\right) K\left(u_{n}(0, s)\right), \quad z=0 \\
u_{n}(z, s)=0, \quad z=-A_{n},
\end{gathered}
$$

with

$$
\begin{gathered}
d_{n}=d\left(z+\zeta_{n}(s)\right), \\
T_{n}=L / \int_{0}^{1} \rho^{-1} R_{0}\left(\zeta_{n}(s)\right) K\left(u_{n}(0, s)\right) d s .
\end{gathered}
$$

We now extend $u_{n}$ to $\Omega$ by

$$
\tilde{u}_{n}= \begin{cases}u_{n} & \text { if } z \in\left[-A_{n}, 0\right] \\ 0 & \text { if } z<-A_{n} .\end{cases}
$$

The estimates for $\tilde{u}_{n}, \tilde{\zeta}_{n}$, and $\widetilde{T}_{n}$ are analogous to those for $u_{m}, \zeta_{m}$, and $T_{m}$. Therefore we can extract a subsequence $\left(\tilde{u}_{n_{k}}, \tilde{\zeta}_{n_{k}}, \widetilde{T}_{n_{k}}\right)$ such that as $k \rightarrow \infty$, we have

$$
\begin{aligned}
\tilde{u}_{n_{k}} & \rightarrow u \quad \text { weakly in } L^{2}(0,1 ; V), \\
\frac{\partial \tilde{u}_{n_{k}}}{\partial z} & \rightarrow \frac{\partial u}{\partial z} \quad \text { weakly in } L^{2}(0,1 ; H), \\
\frac{\partial}{\partial z}\left(d_{n_{k}} \frac{\partial \tilde{u}_{n_{k}}}{\partial z}\right) & \rightarrow \frac{\partial}{\partial z}\left(d \frac{\partial u}{\partial z}\right) \quad \text { weakly in } L^{2}\left(0,1 ; V^{\prime}\right), \\
\frac{\partial \tilde{u}_{n_{k}}}{\partial s} & \rightarrow \frac{\partial u}{\partial s} \quad \text { weakly in } L^{2}\left(0,1 ; V^{\prime}\right),
\end{aligned}
$$

with $d_{n_{k}}=d\left(z+\zeta_{n_{k}}\right), d=d(z+\zeta)$. 
We also have

$$
K\left(\tilde{u}_{n_{k}}(0, \cdot)\right) \rightarrow K(u(0, \cdot)) \quad \text { strongly in } L^{p}(0,1), 1 \leq p<\infty .
$$

In fact, $\tilde{u}_{n}$ bounded in $L^{2}(0,1 ; V)$ implies that $\tilde{u}_{n}$ is also bounded in $L^{2}\left(0,1 ; H^{-1}(-\delta, 0)\right)$ with $0<\delta<A_{n}, \delta$ fixed. Then we proceed, as for the proof of Lemma 4.2 with $\Omega=(-\delta, 0)$, to deduce Eq. (4.65).

As for $\tilde{\zeta}_{n_{k}}$, we have results analogous to those on $\zeta_{m}$, i.e.,

$$
\begin{gathered}
\tilde{\zeta}_{n_{k}} \rightarrow \zeta \quad \text { uniformly on }[0,1], \\
\frac{d \tilde{\zeta}_{n_{k}}}{d s} \rightarrow \frac{d \zeta}{d s} \quad \text { in } L^{\infty}(0,1) \text { weak star. }
\end{gathered}
$$

Finally, denoting the limit of $\widetilde{T}_{n_{k}}$ by $T$, we see that $(u, \zeta, T)$ satisfies Eqs. (4.10)(4.13), the passage to the limit in Eqs. (4.57)-(4.60) being straightforward. This completes the proof of Theorem 4.2.

5. Homogenization process: limit of $u^{\varepsilon}$. In this section, we will show that the periodic solution of the problem (4.1)-(4.3) converges to a travelling wave solution as $\varepsilon$ goes to zero. We first obtain the homogenized equation formally using Ansatz expansions before proceeding with the theoretical proof using energy estimates. Note that the Ansatz will be performed in a moving domain, while the proof will be carried out in a fixed domain.

5.1. Setting up the problem. In addition to the notation of Secs. 2 and 4, we define for $T>0$ fixed

$$
\begin{aligned}
Q & =\Omega \times(0, T), \\
\theta^{\varepsilon} & =\left\{(x, t) \in Q: x<\zeta^{\varepsilon}(t)\right\}, \\
\theta & =\{(x, t) \in Q: x<\zeta(t)\} .
\end{aligned}
$$

Let $L_{\varepsilon}=\varepsilon Y$ be the spatial wavelength. We know that $R_{0}^{\varepsilon}$ and $d^{\varepsilon}$ are $\varepsilon Y$-periodic in $z$ and $u^{\varepsilon}$ is $T^{\varepsilon}$-periodic in time with

$$
\varepsilon Y=\int_{0}^{T^{\varepsilon}} \rho^{-1} R_{0}^{\varepsilon}\left(\zeta^{\varepsilon}(t)\right) K\left(u^{\varepsilon}(0, t)\right) d t .
$$

We then define the microscopic variable $y$ as

$$
y=\frac{x}{\varepsilon}
$$

and consider the two functions $R_{0}=R_{0}(y), d=d(y)$, both periodic in $y$ and bounded.

We next set

$$
R_{0}^{\varepsilon}(x)=R_{0}(x / \varepsilon) \quad \text { and } \quad d^{\varepsilon}(x)=d(x / \varepsilon)
$$

and consider the problem

$$
c \frac{\partial u^{\varepsilon}}{\partial t}-\frac{\partial}{\partial x}\left(d^{\varepsilon}(x) \frac{\partial u^{\varepsilon}}{\partial x}\right)=0, \quad x<\zeta^{\varepsilon}(t)
$$




$$
\frac{d \zeta^{\varepsilon}}{d t}=-\rho^{-1} R_{0}^{\varepsilon}(x) K\left(u^{\varepsilon}(x, t)\right), \quad x=\zeta^{\varepsilon}(t)
$$

with the boundary conditions

$$
\begin{gathered}
d^{\varepsilon} \frac{\partial u^{\varepsilon}}{\partial x}=\theta \beta R_{0} K\left(u^{\varepsilon}\right), \quad x=\zeta^{\varepsilon}(t), \\
u^{\varepsilon}(x, t) \rightarrow 0, \quad x \rightarrow-\infty,
\end{gathered}
$$

and periodic and initial conditions

$$
u^{\varepsilon}(x, 0)=u^{\varepsilon}\left(x, T^{\varepsilon}\right) ; \quad \zeta^{\varepsilon}(0)=0 .
$$

5.2. Asymptotic expansions. By considering $x$ and $y$ as two independent variables in space, we perform the transformation

$$
(x, t) \rightarrow(x, y, t),
$$

corresponding to $y=x / \varepsilon$. We thus have

$$
\frac{\partial}{\partial x}=\frac{1}{\varepsilon} \frac{\partial}{\partial y}+\frac{\partial}{\partial x}
$$

and Eqs. (5.3)-(5.4) become

$$
\begin{array}{cc}
c \frac{\partial u^{\varepsilon}}{\partial t}+A^{\varepsilon} u^{\varepsilon}=0, & x<\zeta^{\varepsilon}(t), \\
\frac{d \zeta^{\varepsilon}}{d t}=-\rho^{-1} R_{0}^{\varepsilon} K\left(u^{\varepsilon}\right), & x=\zeta^{\varepsilon}(t),
\end{array}
$$

with $A^{\varepsilon}=\varepsilon^{-2} A_{1}+\varepsilon^{-1} A_{2}+A_{3}$ and

$$
\begin{aligned}
& A_{1}=-\frac{\partial}{\partial y}\left(d(y) \frac{\partial}{\partial y}\right), \\
& A_{2}=-\frac{\partial}{\partial x}\left(d(y) \frac{\partial}{\partial y}\right)-\frac{\partial}{\partial y}\left(d(y) \frac{\partial}{\partial x}\right), \\
& A_{3}=-d(y) \frac{\partial^{2}}{\partial x^{2}} .
\end{aligned}
$$

We now look for $u^{\varepsilon}$ and $\zeta^{\varepsilon}$ in the form

$$
\begin{gathered}
u^{\varepsilon}(x, y, t)=u_{0}(x, y, t)+\varepsilon u_{1}(x, y, t)+\varepsilon^{2} u_{2}(x, y, t)+\cdots, \\
\zeta^{\varepsilon}(t)=\zeta_{0}(t)+\varepsilon \zeta_{1}(t)+\cdots,
\end{gathered}
$$

with $u_{i} \quad Y$-periodic in $y$.

5.2.1. Homogenization of Eq. (5.10). By identifying powers of $\varepsilon$ in Eq. (5.10), we obtain

$$
\begin{gathered}
A_{1} u_{0}=0 \quad\left(\varepsilon^{-2}\right), \\
A_{1} u_{1}+A_{2} u_{0}=0 \quad\left(\varepsilon^{-1}\right), \\
A_{1} u_{2}+A_{2} u_{1}+A_{3} u_{0}+c \frac{\partial u_{0}}{\partial t}=0 \quad\left(\varepsilon^{0}\right) .
\end{gathered}
$$


From Eq. (5.14), we deduce that

$$
u_{0}=u_{0}(x, t)
$$

Equation (5.15) then reduces to

$$
A_{1} u_{1}=\frac{\partial}{\partial y}\left(d \frac{\partial u_{0}}{\partial x}\right)=-\left(A_{1} y\right) \frac{\partial u_{0}}{\partial x} .
$$

By introducing $J(y)$ as a $Y$-periodic solution of $A_{1} J=A_{1} y$, we write the general solution of Eq. (5.18) as

$$
u_{1}=-J(y) \frac{\partial u_{0}}{\partial x}+\hat{u}_{1}(x, t)
$$

with

$$
J(y)=y-\frac{1}{m_{y}(1 / d)} \int_{0}^{y} \frac{d w}{d(w)}+K, \quad K=\text { const },
$$

where $m_{y}$ represents the mean over one period.

We can now solve Eq. (5.16) for $u_{2}$ iff

$$
m_{y}\left(A_{2} u_{1}+A_{3} u_{0}+c \frac{\partial u_{0}}{\partial t}\right)=0
$$

Since

$$
A_{2} u_{1}=d \frac{d J}{d y} \frac{\partial^{2} u_{0}}{\partial x^{2}}-\frac{\partial}{\partial y}\left(-J d \frac{\partial^{2} u_{0}}{\partial x^{2}}+d \frac{\partial \hat{u}_{1}}{\partial x}\right), \quad A_{3} u_{0}=-d \frac{\partial^{2} u_{0}}{\partial x^{2}},
$$

Eq. (5.21) becomes

$$
m_{y}\left(d \frac{d J}{d y}\right) \frac{\partial^{2} u_{0}}{\partial x^{2}}-m_{y}(d) \frac{\partial^{2} u_{0}}{\partial x^{2}}+c \frac{\partial u_{0}}{\partial t}=0 .
$$

We observe from Eq. (5.20) that

$$
m_{y}\left(d-d \frac{\partial J}{\partial y}\right)=\frac{1}{m_{y}(1 / d)}
$$

so that Eq. (5.22) reduces to

$$
c \frac{\partial u_{0}}{\partial t}-\frac{1}{m_{y}(1 / d)} \frac{\partial^{2} u_{0}}{\partial x^{2}}=0, \quad x<\zeta_{0}(t),
$$

which is the (formal) homogenization of Eq. (5.10).

5.2.2. The homogenized speed. From Eqs. (5.12), (5.13), we have the following expansions:

$$
\begin{gathered}
\frac{d \zeta^{\varepsilon}}{d t}=\frac{d \zeta_{0}}{d t}+\varepsilon \frac{d \zeta_{1}}{d t}+\cdots \\
K\left(u^{\varepsilon}\right)=K\left(u_{0}\right)+\varepsilon K^{\prime}\left(u_{0}\right) u_{1}+\cdots \\
u^{\varepsilon}\left(\zeta^{\varepsilon}\right)=u_{0}\left(\zeta_{0}\right)+\varepsilon\left(u_{0}^{\prime}\left(\zeta_{0}\right) \zeta_{1}+u_{1}\left(\zeta_{0}\right)\right)+\cdots
\end{gathered}
$$

The homogenized speed is then given by

$$
\frac{d \zeta_{0}}{d t}=-\rho^{-1} R_{00} K\left(u_{0}\left(\zeta_{0}(t), t\right)\right)
$$


where $R_{00}$ is the zeroth-order term in a formal expansion of $R_{0}^{\varepsilon}\left(\zeta^{\varepsilon}\right)$. At first sight, it is tempting to believe that

$$
R_{00}=m_{y}\left(R_{0}\right) .
$$

But as we show in Sec. 5.3, $R_{00}$ is in fact the harmonic mean of $R_{0}$, i.e.,

$$
R_{00}=\frac{1}{m_{y}\left(1 / R_{0}\right)} \text {. }
$$

We have

$$
\begin{gathered}
\frac{1}{m_{y}(1 / d)} \frac{\partial u_{0}}{\partial x}=\theta \beta R_{00} K\left(u_{0}(x, t)\right) \text { for } x=\zeta_{0}(t) \\
u_{0}(x, t) \rightarrow 0 \quad \text { as } x \rightarrow-\infty .
\end{gathered}
$$

The complete homogenized system is

$$
\begin{gathered}
c \frac{\partial u_{0}}{\partial t}-\frac{1}{m_{y}(1 / d)} \frac{\partial^{2} u_{0}}{\partial x^{2}}=0, \quad x<\zeta_{0}(t), \\
\frac{d \zeta_{0}}{d t}=-\rho^{-1} R_{00} K\left(u_{0}\left(\zeta_{0}(t), t\right),\right.
\end{gathered}
$$

and the boundary conditions are

$$
\begin{gathered}
\frac{1}{m_{y}(1 / d)} \frac{\partial u_{0}}{\partial x}=\theta \beta R_{00} K\left(u_{0}(x, t)\right), \quad x=\zeta_{0}(t), \\
u_{0}(x, t) \rightarrow 0, \quad x \rightarrow-\infty .
\end{gathered}
$$

5.2.3. A solution of the homogenized system. Recalling that $u^{\varepsilon}$ is periodic in time with period $T^{\varepsilon}$, which tends to zero as $\varepsilon$ goes to zero, it is natural to think that $u^{\varepsilon}$ converges to a stationary wave propagating with a constant velocity $-v$ with $v>0$. Therefore we look for a travelling wave solution of Eqs. (5.32). Let $z=x+v t$; then Eqs. (5.32) become

$$
\begin{gathered}
\frac{1}{m_{y}(1 / d)} \frac{d^{2} u_{0}}{d z^{2}}-c v \frac{d u_{0}}{d z}=0, \quad z<0, \\
\frac{1}{m_{y}(1 / d)} \frac{d u_{0}}{d z}=\theta \beta R_{00} K\left(u_{0}(0)\right), \\
u_{0}(z) \rightarrow 0 \quad \text { as } z \rightarrow-\infty
\end{gathered}
$$

By noting that the speed $v$ is given by

$$
v=\rho^{-1} R_{00} K\left(u_{0}(0)\right),
$$

we find an explicit solution of Eqs. (5.33):

$$
u_{0}(z)=\theta \beta \rho c^{-1} \exp \left(\frac{c v z}{d_{h}}\right), \quad z<0,
$$

where $d_{h}=\frac{1}{m_{y}(1 / d)}$.

In the next section, we justify the formal homogenized results obtained above. 
5.3. Justification of the homogenized equations. In this section, the equations will be considered in the front-fixed frame by using the coordinate transformation $z=$ $x-\zeta^{\varepsilon}(t)$. The homogenization result is stated in the first part as Theorem 5.1, and the rest of this section will be devoted to its proof.

Equations (5.3)-(5.6) in the front-fixed frame appear as

$$
\begin{gathered}
c \frac{\partial u^{\varepsilon}}{\partial t}-c \frac{d \zeta^{\varepsilon}}{d t} \frac{\partial u^{\varepsilon}}{\partial z}-\frac{\partial}{\partial z}\left(d^{\varepsilon}\left(z+\zeta^{\varepsilon}(t)\right) \frac{\partial u^{\varepsilon}}{\partial z}\right)=0, \quad z<0, \\
\frac{d \zeta^{\varepsilon}}{d t}=-\rho^{-1} R_{0}^{\varepsilon}\left(\zeta^{\varepsilon}(t)\right) K\left(u^{\varepsilon}(0, t)\right),
\end{gathered}
$$

subject to the boundary conditions

$$
\begin{gathered}
d^{\varepsilon}\left(\zeta^{\varepsilon}(t)\right) \frac{\partial u^{\varepsilon}}{\partial z}=\theta \beta R_{0}^{\varepsilon}\left(\zeta^{\varepsilon}(t)\right) K\left(u^{\varepsilon}(z, t)\right), \quad z=0, \\
u^{\varepsilon}(z, t) \rightarrow 0 \quad \text { as } z \rightarrow-\infty .
\end{gathered}
$$

5.3.1. Statement of the homogenization result. The notation is that of Sec. 5.1. Theorem 5.1. Let $T>0$ be fixed. Under the assumptions (4.5), (4.6) of Sec. 4.1, we have the convergence results:

$$
\begin{gathered}
u^{\varepsilon} \rightarrow u \text { weakly in } L^{2}(0, T ; V), \\
\zeta^{\varepsilon} \rightarrow \zeta \text { uniformly on }[0, T]
\end{gathered}
$$

as $\varepsilon \rightarrow 0$, with $(u, \zeta)$ satisfying the equations:

$$
\begin{gathered}
c \frac{d \zeta}{d t} \frac{d u}{d z}+\frac{1}{m_{y}(1 / d)} \frac{d^{2} u}{d z^{2}}=0, \quad z<0, \\
\frac{d \zeta}{d t}=-\frac{\rho^{-1}}{m_{y}\left(1 / R_{0}\right)} K(u(0)),
\end{gathered}
$$

subject to the boundary conditions

$$
\begin{gathered}
\frac{1}{m_{y}(1 / d)} \frac{d u}{d z}=\theta \beta \frac{1}{m_{y}\left(1 / R_{0}\right)} K(u(0)), \quad z=0, \\
u(z) \rightarrow 0 \quad \text { as } z \rightarrow-\infty .
\end{gathered}
$$

5.3.2. Proof of Theorem 5.1. We will first show that the limit $u$ is independent of time before proceeding with the proof that $(u, \zeta)$ satisfies Eqs. $(5.40),(5.41)$. We finally derive the homogenized boundary conditions.

The estimates on $u^{\varepsilon}$ and $\zeta^{\varepsilon}$ are analogous to those on $u_{m}$ and $\zeta_{m}$ of Sec. 4 (cf. 4.4.2). Also note that these estimates are the same whether we use the $x$ or $z$ coordinates. Therefore we can extract subsequences, still denoted by $u^{\varepsilon}$ and $\zeta^{\varepsilon}$, such that

$$
\begin{gathered}
u^{\varepsilon} \rightarrow u \quad \text { weakly in } L^{2}(0, T ; V), \\
\zeta^{\varepsilon} \rightarrow \zeta \quad \text { uniformly on }[0, T] .
\end{gathered}
$$


We also have

$$
\begin{gathered}
\frac{\partial u^{\varepsilon}}{\partial t} \rightarrow \frac{\partial u}{\partial t} \quad \text { weakly in } L^{2}\left(0, T ; V^{\prime}\right), \\
\frac{d \zeta^{\varepsilon}}{d t} \rightarrow \frac{d \zeta}{d t} \quad \text { in } L^{\infty}(0, T) \text { weak star. }
\end{gathered}
$$

Note that from Eqs. (5.44), (5.46), we can also write (up to an extraction)

$$
u^{\varepsilon} \rightarrow u \quad \text { strongly in } L^{2}\left(0, T ; L^{2}\left(\Omega^{\prime}\right)\right)
$$

with $\Omega^{\prime}$ a bounded interval of $\Omega$. Therefore the sequence $u^{\varepsilon}$ will be assumed to be strongly $L^{2}$-convergent on the support of any test function of $Q=\Omega \times(0, T)$.

LemmA 5.1. The limit of $u^{\varepsilon}$ is independent of time, i.e., $\partial u / \partial t=0$.

Proof. From Eq. (5.46), this amounts to proving that for $\phi \in L^{2}(0, T ; V)$,

$$
\left\langle\frac{\partial u^{\varepsilon}}{\partial t}, \phi\right\rangle_{L^{2}\left(0, T ; V^{\prime}\right), L^{2}(0, T ; V)}=\int_{0}^{T}\left(\frac{\partial u^{\varepsilon}}{\partial t}, \phi\right)_{V^{\prime}, V} d t \rightarrow 0 \quad \text { as } \varepsilon \rightarrow 0
$$

REMARK. It suffices to take $\phi \equiv v \in V$. In fact, $\phi \in L^{2}(0, T ; V)$ can be approximated by $\phi_{n} \in L^{2}(0, T ; V)$ with $\phi_{n}$ piecewise constant functions in time, so that Eq. (5.49) can be proved on each subinterval.

Now there exists an integer $m$ such that

$$
T=m T^{\varepsilon}+\delta_{\varepsilon}, \quad 0 \leq \delta_{\varepsilon}<T^{\varepsilon},
$$

and $u^{\varepsilon}$ being $T^{\varepsilon}$-periodic in time, we have

$$
\int_{0}^{m T^{\varepsilon}} \frac{\partial u^{\varepsilon}}{\partial t}=0
$$

so that the duality product in Eq. (5.49) reduces to

$$
\left\langle\frac{\partial u^{\varepsilon}}{\partial t}, v\right\rangle=\int_{0}^{\delta_{\varepsilon}}\left(\frac{\partial u^{\varepsilon}}{\partial t}(t), v\right) d t
$$

We then have

$$
\begin{aligned}
\left|\int_{0}^{\delta_{\varepsilon}}\left(\frac{\partial u^{\varepsilon}}{\partial t}(t), v\right)_{V^{\prime}, V} d t\right| & \leq \int_{0}^{\delta_{\varepsilon}}\left\|\frac{\partial u^{\varepsilon}}{\partial t}(t)\right\|_{V^{\prime}}\|v\| d t \\
& \leq\|v\| \int_{0}^{T}\left\|\frac{\partial u^{\varepsilon}}{\partial t}(t)\right\|_{V^{\prime}} \chi_{\left[0, \delta_{\varepsilon}\right]}(t) d t
\end{aligned}
$$

The Cauchy-Schwarz inequality applied to the above integral yields

$$
\int_{0}^{T}\left\|\frac{\partial u^{\varepsilon}}{\partial t}(t)\right\|_{V^{\prime}} \chi_{\left[0, \delta_{\varepsilon}\right]}(t) d t \leq\left\|\frac{\partial u^{\varepsilon}}{\partial t}\right\|_{L^{2}\left(0, T ; V^{\prime}\right)} \sqrt{\delta_{\varepsilon}}
$$

Since $\partial u^{\varepsilon} / \partial t$ is bounded in $L^{2}\left(0, T ; V^{\prime}\right)$, we finally obtain

$$
\left\langle\frac{\partial u^{\varepsilon}}{\partial t}, v\right\rangle \leq C \sqrt{\delta_{\varepsilon}}\|v\|
$$


with $\delta_{\varepsilon} \rightarrow 0$ as $\varepsilon \rightarrow 0$. Thus for all $v \in V$, we have

$$
\left\langle\frac{\partial u^{\varepsilon}}{\partial t}, v\right\rangle \rightarrow 0 \quad \text { as } \varepsilon \rightarrow 0
$$

and this completes the proof of the lemma.

We now proceed by constructing a suitable test function $\psi$. Given $P(y)$ as a first degree polynomial in $y$, we define $w(y)$ as the solution (up to an additive constant) of

$$
A_{1} w=0
$$

with $A_{1}=-\frac{\partial}{\partial y}\left(d(y) \frac{\partial}{\partial y}\right)$ as in Sec. 5.2 and where $w-P$ is a $Y$-periodic function in $y$. We then define

$$
w^{\varepsilon}(x)=\varepsilon w\left(\frac{x}{\varepsilon}\right)
$$

and observe that

$$
\frac{\partial}{\partial x}\left(d^{\varepsilon}(x) \frac{\partial w^{\varepsilon}}{\partial x}\right)=0 .
$$

Equation (5.53), expressed in the variable $z$, becomes

$$
\frac{\partial}{\partial z}\left(d^{\varepsilon}\left(z+\zeta^{\varepsilon}(t)\right) \frac{\partial w^{\varepsilon}}{\partial z}\left(z+\zeta^{\varepsilon}(t)\right)\right)=0, \quad z<0 .
$$

We now set

$$
\hat{w}^{\varepsilon}(z, t)=w^{\varepsilon}\left(z+\zeta^{\varepsilon}(t)\right)
$$

and consider a test function $\phi \in C_{0}^{\infty}(Q)$. We then define $\psi$ as

$$
\psi=\phi \hat{w}^{\varepsilon} \text {, }
$$

and this gives us the required test function. We now multiply Eq. (5.36) by $\psi$ and integrate over $Q$, obtaining

$$
\int_{Q} c\left(\frac{\partial u^{\varepsilon}}{\partial t}-\frac{d \zeta^{\varepsilon}}{\partial t} \frac{\partial u^{\varepsilon}}{\partial z}\right) \phi \hat{w}^{\varepsilon} d z d t-\int_{Q} \frac{\partial}{\partial z}\left(d^{\varepsilon}\left(z+\zeta^{\varepsilon}(t)\right) \frac{\partial u^{\varepsilon}}{\partial z}\right) \phi \hat{w}^{\varepsilon} d z d t=0 .
$$

We next multiply (5.54) by $\phi u^{\varepsilon}$ and integrate over $Q$ to obtain

$$
\int_{Q} \frac{\partial}{\partial z}\left(d^{\varepsilon}\left(z+\zeta^{\varepsilon}(t)\right) \frac{\partial \hat{w}^{\varepsilon}}{\partial z}\right) \phi u^{\varepsilon} d z d t=0
$$

which, after integration by parts, gives

$$
\int_{Q} d^{\varepsilon}\left(z+\zeta^{\varepsilon}(t)\right) \frac{\partial \hat{w}^{\varepsilon}}{\partial z} \frac{\partial w^{\varepsilon}}{\partial z} \phi d z d t+\int_{Q} d^{\varepsilon}\left(z+\zeta^{\varepsilon}(t)\right) \frac{\partial \hat{w}^{\varepsilon}}{\partial z} u^{\varepsilon} \frac{\partial \phi}{\partial z} d z d t=0 .
$$

We now subtract Eq. (5.58) from Eq. (5.57). This gives

$$
\begin{aligned}
\int_{Q} c & \left(\frac{\partial u^{\varepsilon}}{\partial t}-\frac{d \zeta^{\varepsilon}}{d t} \frac{\partial u^{\varepsilon}}{d z}\right) \phi \hat{w}^{\varepsilon} d z d t \\
& =\int_{Q} d^{\varepsilon}\left(z+\zeta^{\varepsilon}(t)\right) \frac{\partial \hat{w}^{\varepsilon}}{\partial z} u^{\varepsilon} \frac{\partial \phi}{\partial z} d z d t-\int_{Q} d^{\varepsilon}\left(z+\zeta^{\varepsilon}(t)\right) \hat{w}^{\varepsilon} \frac{\partial u^{\varepsilon}}{\partial z} \frac{\partial \phi}{\partial z} d z d t .
\end{aligned}
$$

It is in this last equation that we are going to pass to the limit. Note that the presence of $\zeta^{\varepsilon}$ in the above terms is a real inconvenience for the passage to the 
limit. To overcome this difficulty, we will proceed via the initial frame, the overall idea being to put the term $\zeta^{\varepsilon}$ in the test function.

(i) Consider the first term. In the initial frame, it becomes [we revert to the $x$ variable by $\left.x=z+\zeta^{\varepsilon}(t)\right]$

$$
\begin{aligned}
X_{1}^{\varepsilon} & =\int_{Q} c\left(\frac{\partial u^{\varepsilon}}{\partial t}-\frac{d \zeta^{\varepsilon}}{d t} \frac{\partial u^{\varepsilon}}{\partial z}\right) \phi \hat{w}^{\varepsilon} d z d t=\int_{\Theta^{\varepsilon}} c \frac{\partial u^{\varepsilon}}{\partial t} w^{\varepsilon}(x) \phi\left(x-\zeta^{\varepsilon}(t), t\right) d x d t \\
& =-\int_{\boldsymbol{\Theta}^{\varepsilon}} c u^{\varepsilon} w^{\varepsilon}\left(\frac{\partial \phi}{\partial t}\left(x-\zeta^{\varepsilon}(t), t\right)-\frac{d \zeta^{\varepsilon}}{d t} \frac{\partial \phi}{\partial x}\left(x-\zeta^{\varepsilon}(t), t\right)\right) d x d t .
\end{aligned}
$$

The latter integral can be written as

$$
X_{1}^{\varepsilon}=-\int_{Q} c \tilde{u}^{\varepsilon} w^{\varepsilon} \chi_{\boldsymbol{\theta}^{\varepsilon}}\left(\frac{\partial \tilde{\phi}}{\partial t}\left(x-\zeta^{\varepsilon}(t), t\right)-\frac{d \zeta^{\varepsilon}}{d t} \frac{\partial \tilde{\phi}}{\partial x}\left(x-\zeta^{\varepsilon}(t), t\right)\right) d x d t
$$

with

$$
\begin{aligned}
\chi_{\Theta^{\varepsilon}} & = \begin{cases}1 & \text { if }(x, t) \in \Theta^{\varepsilon} \\
0 & \text { otherwise } ;\end{cases} \\
\tilde{u}^{\varepsilon}(x, t) & = \begin{cases}u^{\varepsilon}(x, t), & x<\zeta^{\varepsilon}(t), \\
u^{\varepsilon}\left(\zeta^{\varepsilon}(t), t\right), & \zeta^{\varepsilon}(t) \leq x<0 .\end{cases}
\end{aligned}
$$

The function $w^{\varepsilon}$ need not be extended, since it is valid for all $x$. It is in this reformulated form (5.60) of the first term that we will pass to the limit.

Limit of $\chi_{\boldsymbol{\theta}^{e}}$. As a result of Eq. (5.45), we have

$$
\chi_{\boldsymbol{\theta}^{\varepsilon}} \rightarrow \chi_{\boldsymbol{\theta}} \text { strongly in } L^{P}(Q), 1 \leq p<\infty,
$$

where $\chi_{\Theta}$ is the characteristic function of the set $\Theta=\{x<\zeta(t)\}$.

From Eq. (5.44), we also have

$$
\tilde{u}^{\varepsilon} \rightarrow \tilde{u} \quad \text { weakly in } L^{2}(0, T ; V)
$$

with $\tilde{u}=$ the corresponding extension of $u$. Note that the result, Eq. (5.48), will enable us to have a strong convergence on $\tilde{u}^{\varepsilon}$ in Eq. (5.60), because $Q$ can be replaced by the compact support of $\tilde{\phi}$.

From the definition of $w$ [cf. Eq. (5.51)], we can set $u=-J(y)+P$ with $J(y)$ as in Eq. (5.20). Then from Eq. (5.52), one can easily see that

$$
w^{\varepsilon} \rightarrow P \text { strongly in } L^{2}(\Omega),
$$

so that we also have

$$
\hat{w}^{\varepsilon} \rightarrow \widehat{P} \text { strongly in } L^{2}(Q)
$$

with $\widehat{P}(z, t)=P(z+\zeta(t))$. Therefore in the limit, Eq. (5.60) becomes

$$
X_{1}=-\int_{Q} c \tilde{u} P \chi_{\Theta}\left(\frac{\partial \tilde{\phi}}{\partial t}(x-\zeta(t), t)-\frac{d \zeta}{d t} \frac{\partial \tilde{\phi}}{\partial x}(x-\zeta(t), t)\right) d x d t,
$$

which can be written as

$$
X_{1}=-\int_{\boldsymbol{\Theta}} c u P \frac{\partial}{\partial t} \phi(x-\zeta(t), t) d x d t
$$


We then integrate by parts with respect to $t$ at least in a weak sense and retransform the resulting term in a front-fixed frame [by $z=x-\zeta(t)]$. Note that $u$ is independent of time (Lemma 5.1), so that the limit of the first term reads finally

$$
X_{1}=-\int_{Q} c \frac{\partial u}{\partial z} \frac{d \zeta}{d t} \widehat{P} \phi d z d t
$$

(ii) We now consider the second term in Eq. (5.59), i.e.,

$$
X_{2}^{\varepsilon}=\int_{Q} d^{\varepsilon}\left(z+\zeta^{\varepsilon}(t)\right) \frac{\partial \hat{w}^{\varepsilon}}{\partial z} u^{\varepsilon} \frac{\partial \phi}{\partial z} d z d t
$$

We proceed as with the first term, by passing through the initial frame. We obtain

$$
X_{2}^{\varepsilon}=\int_{Q} \tilde{d}^{\varepsilon}(x) \frac{\partial w^{\varepsilon}}{\partial x} \tilde{u}^{\varepsilon} \frac{\partial \tilde{\phi}}{\partial x}\left(x-\zeta^{\varepsilon}(t)\right) \chi_{\boldsymbol{\theta}^{\varepsilon}} d x d t,
$$

where $\tilde{d}^{\varepsilon}, \tilde{u}^{\varepsilon}$, and $\tilde{\phi}$ are the respective extensions of $d^{\varepsilon}, u^{\varepsilon}$, and $\phi . \tilde{u}^{\varepsilon}$ and $\tilde{\phi}$ are defined as previously and $\tilde{d}^{\varepsilon}$ is obtained by extending $d^{\varepsilon}$ by periodicity up to the origin. To be able to pass to the limit in Eq. (5.67), we must find a limit for $\tilde{d}^{\varepsilon} \partial w^{\varepsilon} / \partial x$. We recall that $d^{\varepsilon}(x)=d(y)$ and from Eq. (5.52) we have $\partial w^{\varepsilon} / \partial x=\partial w / \partial y$. Since $d(y)$ and $\partial w / \partial y$ are $Y$-periodic, bounded functions in $y$, we have

$$
\tilde{d} \frac{\partial w^{\varepsilon}}{\partial x} \rightarrow m_{y}\left(d \frac{\partial w}{\partial y}\right) \text { in } L^{\infty}(\Omega) \text { weak star. }
$$

Therefore the limit of $X_{2}^{\varepsilon}$ as $\varepsilon$ tends to zero is

$$
X_{2}=\int_{Q} m_{y}\left(d \frac{\partial w}{\partial y}\right) \tilde{u} \frac{\partial \tilde{\phi}}{\partial x}(x-\zeta(t), t) \chi_{\Theta} d x d t,
$$

which can be rewritten in the front-fixed frame as

$$
X_{2}=\int_{Q} m_{y}\left(d \frac{\partial w}{\partial y}\right) u(z) \frac{\partial \phi}{\partial z} d z d t
$$

(iii) Now consider finally the last term in Eq. (5.59), i.e.,

$$
X_{3}^{\varepsilon}=\int_{Q} d^{\varepsilon}\left(z+\zeta^{\varepsilon}(t)\right) \hat{w}^{\varepsilon} \frac{\partial u^{\varepsilon}}{\partial z} \frac{\partial \phi}{\partial z} d z d t
$$

We set

$$
\gamma^{\varepsilon}(z, t)=d^{\varepsilon}\left(z+\zeta^{\varepsilon}(t)\right) \frac{\partial u^{\varepsilon}}{\partial z} .
$$

Since $\gamma^{\varepsilon}$ remains in a bounded set in $L^{2}(Q)$, we have, as $\varepsilon$ tends to zero,

$$
\gamma^{\varepsilon} \rightarrow \gamma \quad \text { weakly in } L^{2}(Q),
$$

so that the limit of the last term is

$$
X_{3}=\int_{Q} \gamma \widehat{P} \frac{\partial \phi}{\partial z} d z d t
$$


Therefore the limit of Eq. (5.59) as $\varepsilon \rightarrow 0$ appears as

$$
\begin{aligned}
\int_{Q} c \frac{\partial u}{\partial z} \frac{\partial \zeta}{\partial t} \widehat{P} \phi d z d t & +\int_{Q} m_{y}\left(d \frac{\partial w}{\partial y}\right) u \frac{\partial \phi}{\partial z}(z, t) d z d t \\
& -\int_{Q} \gamma \widehat{P} \frac{\partial \phi}{\partial z} d z d t=0, \quad z<0 .
\end{aligned}
$$

We will use this equation to find what $\gamma$ is equal to. Before proceeding further, however, let us consider the initial equation in $\varepsilon$ [cf. Eq. (5.36)]

$$
c \frac{\partial u^{\varepsilon}}{\partial t}-\frac{\partial \Gamma^{\varepsilon}}{\partial z}=0, \quad z<0,
$$

with $\Gamma^{\varepsilon}=\gamma^{\varepsilon}+c \frac{d \zeta^{\varepsilon}}{d t} u^{\varepsilon}$. In the limit $\varepsilon \rightarrow 0$, we have, thanks to Eq. (5.48),

$$
\Gamma^{\varepsilon} \rightarrow \Gamma=\gamma+c \frac{d \zeta}{d t} u \quad \text { weakly in } L^{2}\left(0, T ; L^{2}\left(\Omega^{\prime}\right)\right) .
$$

Since $\partial \Gamma^{\varepsilon} / \partial z$ remains bounded in $L^{2}\left(0, T ; V^{\prime}\right)$, we can therefore write

$$
\frac{\partial \Gamma^{\varepsilon}}{\partial z} \rightarrow \frac{\partial \Gamma}{\partial z} \quad \text { weakly in } L^{2}\left(0, T ; V^{\prime}\right) \text {, }
$$

so that in the limit, Eq. (5.73) becomes

$$
\frac{\partial \Gamma}{\partial z}=0, \quad x<0
$$

This will give the required homogenized result with $\gamma$ given by

Proposition 5.1.

$$
\gamma=m_{y}\left(d \frac{\partial w}{\partial y}\right) \frac{\partial u}{\partial z}
$$

Proof. In fact, Eq. (5.72) can be reduced to

$$
\int_{Q} \gamma \frac{\partial \widehat{P}}{\partial z} \phi d z d t+\int_{Q} m_{y}\left(d \frac{\partial w}{\partial y}\right) u \frac{\partial \phi}{\partial z} d z d t=0 .
$$

It suffices to integrate the third term by parts with respect to $z$ and use Eq. (5.76) to obtain the required result.

By recalling that $\widehat{P}(z, t)=P(z+\zeta(t))$ and that $P(x)=x$, we have $\partial \widehat{P} / \partial z=1$, so that Eq. (5.78) becomes

$$
\int_{Q}\left(\gamma-m_{y}\left(d \frac{\partial w}{\partial y}\right) \frac{d u}{d z}\right) \phi d z d t=0
$$

for all smooth $\phi$ with compact support in $Q$. We have therefore

$$
\gamma=m_{y}\left(d \frac{\partial w}{\partial y}\right) \frac{d u}{d z}
$$

and this completes the proof of Proposition 5.1.

Now by taking $w(y)=-J(y)+P(y)$ with $P(y)=y$, we have

$$
m_{y}\left(d \frac{\partial W}{\partial y}\right)=m_{y}\left(d-d \frac{\partial J}{\partial y}\right)=\frac{1}{m_{y}(1 / d)},
$$


and thus

$$
\gamma=\frac{1}{m_{y}(1 / d)} \frac{\partial u}{\partial z} .
$$

This leads to the final homogenized equation

$$
c \frac{d \zeta}{d t} \frac{\partial u}{\partial z}+\frac{1}{m_{y}(1 / d)} \frac{\partial^{2} u}{\partial z^{2}}=0, \quad z<0 .
$$

Now we still have to find the homogenized velocity $d \zeta / d t$ in Eq. (5.82). We state the following result.

Proposition 5.2.

$$
\frac{d \zeta}{d t}=-\frac{\rho^{-1}}{m_{y}\left(1 / R_{0}\right)} K(u(0)) .
$$

Proof. We recall that the velocity is given by

$$
\frac{d \zeta^{\varepsilon}}{d t}=-\rho^{-1} R_{0}^{\varepsilon}\left(\zeta^{\varepsilon}(t)\right) K\left(u^{\varepsilon}(0, t)\right) .
$$

From Lemma 4.2, we have

$$
K\left(u^{\varepsilon}(0, \cdot)\right) \rightarrow K(u(0)) \quad \text { strongly in } L^{p}(0, T), \quad 1 \leq p<\infty .
$$

By dividing on both sides by $R_{0}^{\varepsilon}\left(\zeta^{\varepsilon}(t)\right)$, we see that Eq. (5.84) becomes

$$
\frac{1}{R_{0}^{\varepsilon}\left(\zeta^{\varepsilon}(t)\right)} \frac{d \zeta^{\varepsilon}}{d t}=-\rho^{-1} K\left(u^{\varepsilon}(0, t)\right)
$$

Let $\psi$ be a test function on $[0, T]$. Then multiply the first term by $\psi$ and integrate over $[0, T]$. We obtain

$$
X_{\varepsilon}=\int_{0}^{T} \frac{1}{R_{0}^{\varepsilon}\left(\zeta^{\varepsilon}(t)\right)} \frac{d \zeta^{\varepsilon}}{d t} \psi d t=0 .
$$

Now we perform the following change of variable:

$$
u=\zeta^{\varepsilon}(t)
$$

Equation (5.86) becomes

$$
X_{\varepsilon}=\int_{0}^{\zeta^{\varepsilon}(T)} \frac{1}{R_{0}^{\varepsilon}(u)} \psi\left(\left(\zeta^{\varepsilon}\right)^{-1}(u)\right) d u .
$$

Using the facts that

$$
\frac{1}{R_{0}{ }^{\varepsilon}} \rightarrow m_{y}\left(1 / R_{0}(y)\right) \text { in } L^{\infty}(\Omega) \text { weak star }
$$

and

$$
\zeta^{\varepsilon} \rightarrow \zeta \quad \text { uniformly on }[0, T]
$$

one can easily show that

$$
X_{\varepsilon} \rightarrow X_{0}=\int_{0}^{\zeta(T)} m_{y}\left(1 / R_{0}(y)\right) \psi\left(\zeta^{-1}(u)\right) d u
$$


By the inverse change of variable $t=\zeta^{-1}(u)$, we have

$$
X_{0}=\int_{0}^{T} m_{y}\left(1 / R_{0}(y)\right) \frac{d \zeta}{d t} \psi(t) d t
$$

and finally

$$
m_{y}\left(1 / R_{0}(y)\right) \frac{d \zeta}{d t}=-\rho^{-1} K(u(0)) .
$$

This completes the proof of Proposition 5.2. Now it remains to derive the homogenized boundary condition.

The homogenized b.c. Let us come back to the initial problem in $\varepsilon$. We have

$$
\begin{gathered}
c \frac{\partial u^{\varepsilon}}{\partial t}-\frac{\partial \Gamma^{\varepsilon}}{\partial z}=0, \quad z<0, \\
d^{\varepsilon}\left(\zeta^{\varepsilon}(t)\right) \frac{\partial u^{\varepsilon}}{\partial z}=\theta \beta R_{0}^{\varepsilon}\left(\zeta^{\varepsilon}(t)\right) K\left(u^{\varepsilon}(0, t)\right), \quad z=0 \\
u^{\varepsilon}(z, t) \rightarrow 0 \quad \text { as } z \rightarrow-\infty .
\end{gathered}
$$

Let $\phi \in L^{2}(0, T ; V)$. Then multiply Eq. (5.90) by $\phi$ and integrate by parts with respect to $z$ over $Q$. We obtain

$$
\int_{Q} c \frac{\partial u^{\varepsilon}}{\partial t} \phi d z d t+\int_{Q} \Gamma^{\varepsilon} \frac{\partial \phi}{\partial z} d z d t-\int_{0}^{T} \Gamma^{\varepsilon}(0, t) \phi(0, t) d t=0 .
$$

Since

$$
\begin{aligned}
\Gamma^{\varepsilon}(0, t) & =d^{\varepsilon}\left(\zeta^{\varepsilon}(t)\right) \frac{\partial u^{\varepsilon}}{\partial z}(0, t)+c \frac{d \zeta^{\varepsilon}}{d t} u^{\varepsilon}(0, t) \\
& =\theta \beta R_{0}^{\varepsilon}\left(\zeta^{\varepsilon}(t)\right) K\left(u^{\varepsilon}(0, t)\right)+c \frac{d \zeta^{\varepsilon}}{d t} u^{\varepsilon}(0, t) \quad \text { [cf. Eq. (5.91)] }
\end{aligned}
$$

Eq. (5.93) becomes

$$
\int_{Q} c \frac{\partial u^{\varepsilon}}{\partial t}+\int_{Q} \Gamma^{\varepsilon} \frac{\partial \phi}{\partial t} d z d t=\int_{0}^{T}\left(\theta \beta R_{0}^{\varepsilon}\left(\zeta^{\varepsilon}(t)\right) K\left(u^{\varepsilon}(0, t)\right)+c \frac{d \zeta^{\varepsilon}}{d t} u^{\varepsilon}(0, t)\right) \phi(0, t) d t
$$

Now we pass to the limit in Eq. (5.94) as $\varepsilon \rightarrow 0$. We obtain

$$
\int_{Q} \Gamma \frac{\partial \phi}{\partial z} d z d t=\int_{0}^{T}\left(\theta \beta \frac{1}{m_{y}\left(1 / R_{0}(y)\right)} K(u(0))+c \frac{d \zeta}{d t} u(0)\right) \phi(0, t) d t .
$$

The first term of Eq. (5.95), when integrated by parts with respect to $z$, gives

$$
-\int_{Q} \frac{\partial \Gamma}{\partial z} \phi d z d t+\int_{0}^{T} \Gamma(0) \phi(0, t) d t
$$

with $\partial \Gamma / \partial z=0$, so that Eq. (5.95) reduces to

$$
\int_{0}^{T} \frac{1}{m_{y}(1 / d(y))} \frac{\partial u}{\partial z}(0) \phi(0, t) d t=\int_{0}^{T} \theta \beta \frac{1}{m_{y}\left(1 / R_{0}(y)\right)} K(u(0)) \phi(0, t) d t
$$

for all $\phi \in L^{2}(0, T ; V)$. Thus we have

$$
\frac{1}{m_{y}(1 / d(y))} \frac{\partial u}{\partial z}(0)=\theta \beta \frac{1}{m_{y}\left(1 / R_{0}(y)\right)} K(u(0)),
$$


which is the homogenized boundary condition at the front.

The final homogenized system in a front-fixed frame is thus given by

$$
\begin{array}{ll}
c \frac{d \zeta}{d t} \frac{d u}{d z}+\frac{1}{m_{y}(1 / d(y))} \frac{d^{2} u}{d z^{2}}=0, & z<0, \\
\frac{d \zeta}{d t}=-\rho^{-1} \frac{1}{m_{y}\left(1 / R_{0}(y)\right)} K(u(0)), & z=0,
\end{array}
$$

with the boundary conditions

$$
\begin{gathered}
\frac{1}{m_{y}(1 / d(y))} \frac{d u}{d z}=\theta \beta \frac{1}{m_{y}\left(1 / R_{0}(y)\right)} K(u), \quad z=0, \\
u(z) \rightarrow 0 \quad \text { as } z \rightarrow-\infty .
\end{gathered}
$$

This completes the proof of Theorem 5.1.

6. Discussion. The model problem considered refers to the steady combustion of a solid reactant with certain properties, which vary in a periodic fashion with respect to distance normal to its combusting surface. If there were no such inhomogeneity, our solution would be a travelling wave with velocity

$$
v=R_{0} K(\theta \beta \rho / c)
$$

and profile

$$
u(z)=\frac{\theta \beta \rho}{c} \exp (c v z / d), \quad z<0,
$$

$z=x+v t$. This is, of course, a $t$-independent temperature distribution in the frame fixed to the combustion front.

If $R_{0}$ and $d$ are allowed to vary in the manner indicated, then we obtain a temperature distribution, which is $t$-periodic in the frame attached to the front, which itself moves with periodic velocity. The existence of a periodic solution was proved.

Finally, we considered the homogenized limit when the wavelength of the striations approaches 0 . It was proved that the periodic solutions approach a $t$-independent profile of the same form as $(6.2)$, but with revised values $\left(v^{\prime}, d^{\prime}\right)$ for the parameters $v$ and $d$. Moreover, in the limit, the front moves with velocity $v^{\prime}$ given by (6.1) with a revised value $R_{0}^{\prime}$ for $R_{0}$. The new parameters $\left(d^{\prime}, R_{0}^{\prime}\right)$ are the harmonic means of the heterogeneous ones:

$$
d^{\prime}=\left(\text { spatial average of } d^{-1}\right)^{-1},
$$

with the same for $R_{0}^{\prime}$.

Since the harmonic mean of a positive periodic function is always less than its average, we find that the homogenized velocity $v^{\prime}$ is less than one would expect from using (6.1) with $R_{0}$ replaced by its average.

It is hoped that the framework set forth here will be suggestive and helpful in future research into the combustion of heterogeneous reactive solids.

Acknowledgments. We thank Dr. B. Gossant and G. Uhrig for helpful discussions and comments. Part of the work was done while P. Fife held a visiting position at the University of Bordeaux I, for which he extends thanks to the university. 


\section{REFERENCES}

[1] M. Artola, Lectures on homogenization given at the University of Bordeaux I, 1986-1987

[2] A. Bensoussan, J. L. Lions, and G. Papanicolaou, Asymptotic analysis for periodic structures, North-Holland, Amsterdam, 1978

[3] C. M. Brauner and Cl. Schmidt-Lainé, Existence of a solution to a certain plane premixed flame with 2-step kinetics, SIAM J. Math. Anal. 18, 1987

[4] R. E. Edwards, Functional Analysis-Theory and Applications, Holt, Rinehart, and Winston, New York, 1965

[5] J. L. Lions, Quelques méthodes de résolution des problèmes aux limites non linéaires, Dunod, Paris, 1969

[6] J. L. Lions and E. Magenes, Problèmes aux limites non homogènes et applications, Vols. 1 et 2, Dunod, Paris, 1968 